\title{
A Dynamic Epistemic Logic with a Knowability Principle
}

\author{
Michael Cohen \\ Munich Center for Mathematical Philosophy, LMU \\ michaelco13@gmail.com
}

\begin{abstract}
A dynamic epistemic logic is presented in which the single agent can reason about his knowledge stages before and after announcements. The logic is generated by reinterpreting multi agent private announcements in a single agent environment. It is shown that a knowability principle is valid for such logic: any initially true $\varphi$ can be known after a certain number of announcements.
\end{abstract}

In recent years a novel explication of knowability has been studied in the framework of dynamic epistemic logic (DEL) 4], in which 'knowable' is read as 'can be known after an announcement' 1, [5]. Under this explication it has been shown that the knowability principle (KP), the principle according to which all truths are knowable, does not hold in public announcement logic (PAL) 1] nor in its extension arbitrary public announcement logic (APAL) [5]. Instead of using public announcements, this contribution focuses on the investigation of knowability using the logical structure of private announcements [4. For this purpose, private announcements are reinterpreted in a single agent environment.

\section{Agents in Private Announcements as Stages of Knowledge}

One can reinterpret the logical structure of private announcements to a set of epistemic agents as an update of a single agent who can reason about past and present stages of knowledge.

For instance, consider the situation in which two agents, 0 and 1, don't know $p$, and the private announcement of $p$ which is given to agent 1 . Let $[\mathcal{E}, e]$ denote this action of private announcement. Then $\mathcal{M}, w \models[\mathcal{E}, e] K_{1}\left(p \wedge \neg K_{0} p\right)$ is the case, which says that after the private announcement, agent 1 knows $p$ and she also knows that agent 0 does not know $p$. This situation can be reinterpreted as one containing a single agent with two sequential stages of knowledge, stage 0 as the initial stage, and stage 1 as the stage after the first announcement. Then $K_{0}$ and $K_{1}$ represent the knowledge stages before and after the first announcement, respectively. Under this interpretation, $[\mathcal{E}, e] K_{1}\left(p \wedge \neg K_{0} p\right)$ is read 'after the first update, the agent knows $\left(K_{1}\right)$ that $p$ and that before the update she didn't know $\left(\neg K_{0}\right) p$ '. The above example is depicted in Fig. 1. For a proper exposition of private announcements in DEL, see ([4], p. 173). 

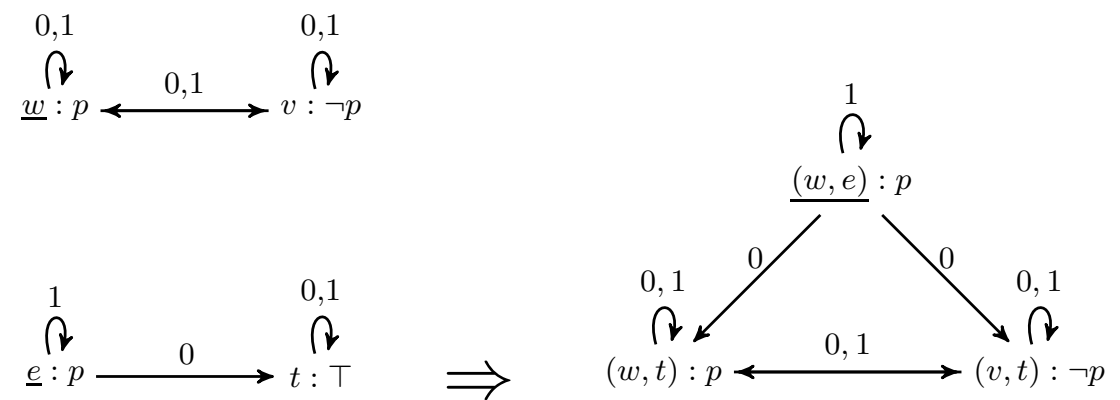

Fig. 1. An example of a private announcement to agent 1 in action model logic (AML). The upper left epistemic model $\mathcal{M}$ describes the initial ignorance of agents 0 and 1 ; the lower left model $\mathcal{E}$ describes the private announcement of $p$ to 1 ; the right model $\mathcal{M} \times \mathcal{E}$ is the epistemic model after the announcement. 0 and 1 can be reinterpreted as stages of knowledge of a single agent.

The aim below is to present such a dynamic epistemic logic of stages of knowledge, as a modification of PAL.

\section{Stages of Knowledge Logic SKL}

The alphabet of stages of knowledge logic (SKL) is the same as of PAL. For the inductive definition of the language of SKL $\mathcal{L}_{S K L}$, we have the following restricted form of a PAL language

$$
\begin{gathered}
\varphi:=|p| \neg \varphi|\varphi \wedge \psi| K_{i} \varphi \mid[\alpha] \psi \text { s.t. } \\
\alpha:=|p| \neg \alpha\left|\alpha_{1} \wedge \alpha_{2}\right| K_{i} \alpha \mid \alpha_{1} \wedge\left[\alpha_{1}\right] \alpha_{2}
\end{gathered}
$$

Let $\mathcal{L}_{E L}$ and $\mathcal{L}_{P L}$ denote the languages of epistemic logic without announcements and of propositional logic, respectively. In SKL, the $K_{i}$ operator represents the single agent's knowledge after the $i$-th announcement. For that we define a degree function $d$ that assigns a natural number to each occurrence of $[\varphi] \psi$ in an SKL formula, s.t. $d([\varphi] \psi)=i$ is read as ' $\varphi$ is the $i$ th announcement'. When clear by context, instead of writing $d([\varphi] \psi)$, we write $d(\varphi)$.

Definition 1. Let $d: \mathcal{L}_{S K L} \rightarrow \mathbb{N}$, be a degree function, assigning every formula and sub-formula in $\mathcal{L}_{S K L}$ a natural number. Let $d_{\text {max }}(\alpha)$ assign for each $\alpha$ of the language the highest $d$ of its sub-formulae. To determine the $d$ of $\alpha \in \mathcal{L}_{S K L}$ and all of its sub-formulae, one applies the following tree rules, where at each node $d$ is applied according to the below specification. For the root of the tree let $n=0$.
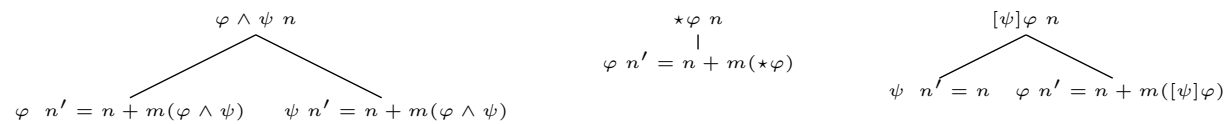


$$
\begin{aligned}
& \text { Where } \left.\star=\neg, K_{i}\right) \\
& d(\alpha)=n+m(\alpha) . m(\alpha)=0 \text { if } \alpha \neq[\varphi] \psi \text {. Otherwise, } m([\varphi] \psi)=1+d_{\max }(\varphi)
\end{aligned}
$$

Definition 2. Given an announcement $[\varphi] \psi$ s.t. $d(\varphi)=n$ define its $\varphi$-list to be a list $\left\langle\alpha_{1}^{\prime} \ldots \varphi\right\rangle$ s.t. the $i$-th member $(1 \leq i \leq n)$ of the list is the announcement of degree $i$ that has, or is within an announcement that has, $\psi$ in its scope. Given $a \varphi$-list, $a \varphi$-sequence is obtained by replacing any member $\alpha_{i}$ in the $\varphi$-list of the form $\beta \wedge[\beta] \gamma$ to $\gamma$ s.t. the $\varphi$ sequence contains only formulae of epistemic logic.

The semantics of SKL can be seen as a modification of PAL semantics (for an exposition of the latter, see 4]). Given a model $\mathcal{M}$, after the announcement of $\varphi$, instead of moving to the model $\mathcal{M}_{\mid \varphi}$ in which all the $\varphi$ states are eliminated as we do in PAL, in SKL we move to a certain union of the existing model $\mathcal{M}$ and its PAL update $\mathcal{M}_{\mid \varphi}$. This requires defining an initial SKL model, its PAL update model, and the union of the two.

Definition 3. Given a single agent $S 5$ epistemic model $\mathcal{M}=(W, R, V)$ its $S K L$ initial model is a structure $\mathcal{M}_{0}=\left\langle W^{0},\left\{R_{i}^{0} \mid i \leq n\right\}, V^{0}\right\rangle$, where:

$W^{0}=\{(w, 0) \mid w \in W\}$.

$R_{i}^{O}=R$ for any $0 \leq i \leq n$.

$(w, 0) \in V^{0}(p)$ iff $w \in V(p)$.

Definition 4. Given a model $\mathcal{M}_{i}$ and an announcement $\varphi$ s.t. $d(\varphi)=i+1$, the PAL' model of $\mathcal{M}_{i}$, written $\mathcal{M}_{i \mid \varphi}$ is:

$W_{\mid \varphi}^{i}=\left\{(w, i+1): w \in W\right.$ and $\left.\mathcal{M}_{i},(w, i) \models \varphi\right\}$

$(w, i+1) R_{j \mid \varphi}^{i}(v, i+1)$ iff $j \geq d(\varphi)$ and $(w, i) R_{j}^{i}(v, i)$

$(w, i+1) \in V_{\mid \varphi}^{i}(p)$ iff $w \in V(p)$.

We abbreviate $(w, i)$ as $w_{i}$.

Definition 5. Given an initial $\mathcal{M}_{0}$ model and an announcement $\varphi$ s.t. $d(\varphi)=n$ and $\left\langle\alpha_{1} \ldots \alpha_{n}\right\rangle$ is the sequence of $\varphi$, define the model $\mathcal{M}_{n}$ to be:

$W^{n}=W^{n-1} \cup W_{\mid \alpha_{n}}^{n-1}$

$R_{j}^{n}=R_{j}^{n-1} \cup R_{j \mid \alpha_{n}}^{n-1}$ and if $j<n$, then $w_{n} R_{j}^{n} v_{j}$ iff $w_{j} R_{j}^{j} v_{j}$

$V^{n}=V^{n-1} \cup V_{\mid \alpha_{n}}^{n-1}$

We read $w_{x} R_{i}^{j} u_{y}$ as 'in the model $\mathcal{M}_{j}, w_{x}$ is related to $u_{y}$ with relation $i$ '. In the $R$ clause of the definition we specify that a state $w_{n}$ can 'look down' at a state $u_{j}(j<n)$ only with the relation $j: w_{n} R_{j}^{n} u_{j}$.

Definition 6. Given an $S K L$ model $\mathcal{M}_{i}$ we define satisfaction as usual with the following change

$\mathcal{M}_{i}, w_{j}=[\varphi] \psi$ iff, if $\mathcal{M}_{i}, w_{j} \models \varphi$, then $\mathcal{M}_{d(\varphi)}, w_{d(\varphi)} \models \psi$

$\mathcal{M}_{i}, w_{j} \models\langle\varphi\rangle \psi$ iff $\mathcal{M}_{i}, w_{j} \models \varphi$, and $\mathcal{M}_{d(\varphi)}, w_{d(\varphi)} \models \psi$

For a simple example of the SKL semantics, consider an agent who initially does not know $p$. After announcing $p$ the agent knows $p$ and that before the 
announcement he didn't know $p: \mathcal{M}_{0}, w_{0} \models[p] K_{1}\left(p \wedge \neg K_{0} p\right)$. This update is depicted in Fig. 2. Note that $d(p)=1$. As was implied earlier, SKL can be reinterpreted as a multi agent logic with $n$ agents s.t. the first announcement is given to agents 1 to $n$ and excludes agent 0 , the second is given to agents 2 to $n$ and excludes agents 0 and 1 , and so on. Similarly to PAL, SKL contains reduction axioms for announcements which allow the translation of each SKL formula to an epistemic logic formula, and which make the system complete [3].

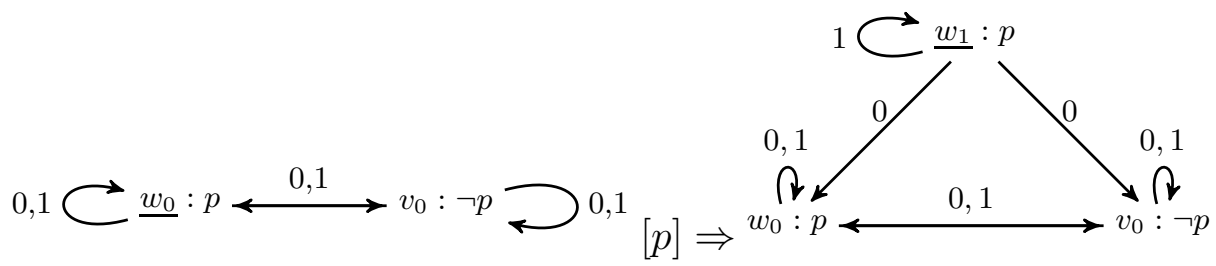

Fig. 2. The single agent who initially does not know $p$ learns $p$. The left model is $\mathcal{M}_{0}$, the initial model. The right model is $\mathcal{M}_{1}$, the model after the first announcement. Note that in $\mathcal{M}_{1}$ relation $R_{1}$ is reflexive. In general, $R_{i}$ is always reflexive in $\mathcal{M}_{i}[3]$.

\section{SKL and the Knowability Principle}

In the philosophical literature the $\mathrm{KP}$ is regularly formulated as $\varphi \rightarrow \diamond K \varphi$. The knowability paradox connected to this formulation is the modal derivation showing that if the KP holds, then all truths are actually known: $\varphi \rightarrow K \varphi[2$. The KP in PAL is formulated as $\varphi \rightarrow\langle\psi\rangle K \varphi$ for some $\psi$, and it is invalid in the latter logic and in its relevant extensions [1, [5]. In this section, we show that for any $\varphi$, the formula $\varphi \rightarrow\langle\psi\rangle K_{n} \varphi$ is true for a 'high enough' $n$. To prove so, we first define the notion of bisimilarity (relevant to SKL).

Definition 7. A non-empty relation $Z \subseteq W^{n} \times W^{m}$ is called a bisimulation from 0 to $k$ between $\mathcal{M}_{n}$ and $\mathcal{M}_{m}$ iff the following conditions are satisfied:

Atoms: If $w_{i} Z w_{j}$ then $w_{i}$ and $v_{j}$ satisfy the same propositional letters.

Forth: If $w_{i} Z w_{j}$ and $w_{i} R_{l}^{n} v_{h}($ s.t. $l \leq k)$, then there is a $v_{x} \in W^{m}$ s.t. $v_{h} Z v_{x}$ and $w_{j} R_{l}^{m} v_{x}$.

Back: If $w_{i} Z w_{j}$ and $w_{j} R_{l}^{m} v_{h}($ s.t. $l \leq k)$, then there is a $v_{x} \in W^{n}$ s.t. $v_{x} Z v_{h}$ and $w_{i} R_{l}^{n} v_{x}$. We write $\left(\mathcal{M}_{n}, w_{i}\right) \leftrightarrows\left(\mathcal{M}_{m}, w_{j}\right)$ for bisimilar states. If $\left(\mathcal{M}_{n}, w_{i}\right) \leftrightarrows$ $\left(\mathcal{M}_{m}, w_{j}\right)$ from 0 to $k$, then $\mathcal{M}_{n}, w_{i}=\varphi$ iff $\mathcal{M}_{m}, w_{j}=\varphi$ for any $\varphi \in \mathcal{L}_{E L}$ with epistemic modalities $K_{0} \ldots K_{k}$.

Lemma 1. $\left(\mathcal{M}_{n}, w_{n}\right) \leftrightarrows\left(\mathcal{M}_{n+1}, w_{n+1}\right)$ from 0 to $n$.

proof sketch: Define a relation $Z \subseteq W^{n} \times W^{n+1}$ s.t. for any $w_{i}$ in $W^{n}$ and $W^{n+1}(i \leq n)$ respectively, $w_{i} Z w_{i}$, and for $w_{n+1} \in W^{n+1}$, let $w_{n} Z w_{n+1}$. Then $Z$ is bisimulation for the modalities $K_{0} \ldots K_{n}$. For a full proof, see the extended version of this contribution ([3], p. 61).

Given the bisimilarity result one can show that a KP is valid in SKL. 
Theorem 1. For an arbitrary initial $S K L$ model $\left(\mathcal{M}_{0}, w_{0}\right)$,

$$
\mathcal{M}_{0}, w_{0} \models \varphi \rightarrow\langle\psi\rangle K_{n} \varphi
$$

for some $\psi$ and $a K_{n}$ s.t. $n \geq 1$.

Proof. The proof goes by the construction of a formula $\psi$ and stage $n$ for each given $\varphi$ of the language of SKL. If $\varphi \in \mathcal{L}_{P L}$ then let $\varphi=\psi$ and $n=1$. Then for such $\varphi, \mathcal{M}_{0}, w_{0} \models \varphi \rightarrow\langle\varphi\rangle K_{1} \varphi$ can be easily checked to be true. Otherwise, assume $\varphi^{\prime} \in \mathcal{L}_{S K L}$ s.t. the highest knowledge modality $K_{m}$ in $\varphi^{\prime}$ is $m$. Start by translating $\varphi^{\prime}$ using the SKL announcement axioms to a formula $\varphi \in \mathcal{L}_{E L}$. Let $\psi$ be identical to a sequence of $m$ announcements of $T$ followed by an announcement of $\varphi$, i.e. $\psi=\langle T\rangle \ldots\langle T\rangle\langle\varphi\rangle$ s.t. $d(\varphi)=m+1$. Consider the following $\mathrm{KP}$ for $\varphi: \mathcal{M}_{0}, w_{0} \models \varphi \rightarrow\langle T\rangle \ldots\langle T\rangle\langle\varphi\rangle K_{m+1} \varphi$. Assume $\mathcal{M}_{0}, w_{0} \models \varphi$. Then $\mathcal{M}_{m} w_{m}=\varphi$ as the announcements of $T$ do not change the truth value of $\varphi$ [3]. Hence, in order to show that $\mathcal{M}_{m}, w_{m} \models\langle\varphi\rangle K_{m+1} \varphi$ it remains to show that $\mathcal{M}_{m+1}, w_{m+1} \models K_{m+1} \varphi$. Pick an arbitrary $u_{x}$ s.t. $w_{m+1} R_{m+1}^{m+1} u_{x}$. By Definition 5 it must be that $u_{x}=u_{m+1}$ and by the assumption of the existence of $u_{m+1}$ it follows that $\mathcal{M}_{m}, u_{m} \models \varphi$. By Lemma $1\left(\mathcal{M}_{m}, u_{m}\right) \leftrightarrow\left(\mathcal{M}_{m+1}, u_{m+1}\right)$ from 0 to $m$, and since by assumption $\varphi$ contains modalities up to $m$, it follows that $\mathcal{M}_{m+1}, u_{m+1} \models \varphi$. Therefore, $\mathcal{M}_{m+1}, w_{m+1} \models K_{m+1} \varphi$ and so $\mathcal{M}_{m}, w_{m} \models$ $\langle\varphi\rangle K_{m+1} \varphi$. Note that sequence of announcements $\langle T\rangle \ldots\langle T\rangle\langle\varphi\rangle$ is equivalent to one nested announcement $\left\langle\psi^{\prime}\right\rangle$ in SKL as in PAL.

Unlike other epistemic logics that can express a KP, SKL avoids the knowability paradox: all truths are knowable but not all truths are known. I note that while the standard exposition of the paradox assumes the necessitation rule for the possibility operator $\diamond$ to derive the paradox [2], the necessitation rule for announcements is unsound in SKL [3].

\section{References}

1. van Benthem, J.: What one come to know. Analysis 64(282), 95-105 (2004)

2. Brogaard, B., Salerno, J.: Fitch's Paradox of Knowability. In: Zalta, E.N. (ed.) The Stanford Encyclopedia of Philosophy (2013), http://plato. stanford.edu/archives/win2013/entries/fitch-paradox

3. Cohen, M.: Dynamic Knowability: The Knowability Paradox in Dynamic Epistemic logic. MA Thesis, LMU Munich (2015)

4. van Ditmarsch, H.P., van der Hoek, W., Kooi, B.: Dynamic Epistemic Logic. Springer, Heidelberg (2007)

5. van Ditmarsch, H.P., van der Hoek, W., Iliev, P.: Everything is knowable - How to Get to Know Whether a Proposition Is True. Theoria 78(2), 93-114 (2012) 